\title{
1 Theta phase mediates deliberate action switching in human 2 Supplementary Motor Areas
}

4 Giovanni Maffei ${ }^{1,2,}$, Riccardo Zucca1,3, Jordi-Ysard Puigbò1,2, Diogo Santos-Pata1, Marco

5 Galli1 ${ }^{1}$, Adrià Tauste Campo ${ }^{2}$, Rodrigo Rocamora ${ }^{2,3}$, Paul Verschure ${ }^{1,4, *}$

71 SPECS Lab., Institute of Bioengineering of Catalonia (IBEC), Barcelona Institute of Science and Technology

8 (BIST), Barcelona, Spain

92 Universitat Pompeu Fabra (UPF), Barcelona, Spain

$10{ }^{3}$ Epilepsy Monitoring Unit, Department of Neurology, Hospital del Mar Medical Research Institute, Barcelona,

11 Spain

$12{ }^{4}$ Institucio' Catalana de Recerca i Estudis Avanc, ats (ICREA), Barcelona, Spain

13 * Corresponding authors: giovanni.maffei@upf.edu, pverschure@ibecbarcelona.eu

\section{ABSTRACT}

17 The ability to deliberately overwrite ongoing automatic actions is a necessary feature of adaptive 18 behavior. It has been proposed that the supplementary motor areas (SMAs) operate as a controller 19 that orchestrates the switching between automatic and deliberate processes by inhibiting ongoing 20 behaviors and so facilitating the execution of alternative ones. In addition, previous studies support 21 the involvement of SMAs theta waves (4-9 Hz) in cognitive control. However, the exact role of such 22 oscillatory dynamics and their contribution to the control of action are not fully understood. To 23 investigate the mechanisms by which the SMAs support direct control of deliberate behavior, we 24 recorded intracranial electroencephalography (iEEG) activity in humans performing a motor 25 sequence task. Subjects had to perform a "change of plans" motor task requiring habitual 26 movements to be overwritten at unpredictable moments. We found that SMAs were exclusively 27 active during trials that demand action reprogramming in response to the unexpected cue but were 28 silent during automatic action execution. Importantly, SMAs activity was characterized by a distinct 29 temporal pattern, expressed in a stereotypical phase alignment of theta oscillations. More 30 specifically, single trial motor performance was correlated with the trial contribution to the global 31 inter-trial phase coherence, with higher coherence associated with faster trials. In addition, theta 32 phase modulated the amplitude of gamma oscillations, with higher cross-frequency coupling in faster 33 trials. Our results suggest that within frontal cortical networks, theta oscillations could encode a 34 control signal that promotes the execution of deliberate actions. 
The ability to deliberately interrupt and overwrite ongoing actions as a response to external cues is a necessary feature of cognitive control. For example, when driving a car on a known route, we automatically perform highly trained actions to reach a familiar destination. However, if the habitual route is found blocked, the automatic driving routines are promptly interrupted, and a new motor program is deliberately assembled and selected to follow an alternative path to the goal.

43 The Supplementary Motor Areas (SMAs), in the Medial Frontal Cortex (MFC), are thought to mediate

44 the switch from automatic to deliberate control when a conflict between current and expected 45 contingencies is detected which requires alterations in ongoing actions (1-5). In particular, it has 46 been suggested that the SMAs orchestrate the balancing of automatic and deliberate processes by 47 inhibiting ongoing motor routines to facilitate the execution of alternative deliberate actions $(4,5)$. 48 Animal studies support this hypothesis, for instance, by showing the involvement of neurons in the 49 primate SMAs in either suppressing or promoting action during "change of plans" paradigms (6). 50 Nevertheless, the functional role of SMAs in humans is still under debate. Imaging studies have 51 reported increased activation of the human pre-SMAs and SMAs during action reprogramming but 52 not during automatic action execution (1). This is consistent with rare lesion studies where subjects 53 suffering from focal SMAs damage are able to stop an action but unable to switch between automatic 54 and deliberate control (7). These results suggest that human SMAs are not generally involved in 55 action inhibition but are specifically supporting switching behavior. However, this interpretation 56 seems at odds with findings from human EEG studies showing significant activation of the medial 57 frontal cortex (MFC) during a behavioral conflict. Here, a positive relationship between the power 58 increase in the theta range (4-8 Hz) and response time during high-conflict trials suggests that this 59 frequency band (often termed 'frontal theta') could reflect a generic inhibitory mechanism, possibly 60 acting as a "global brake" on the motor system both pro- and retrospectively (i.e. post-error slowing) 61 (8-10). Hence, the exact role of human SMAs in cognitive control is not clear and pointing to a 62 plurality of possible functions.

63 A second topic of debate pertains to the mechanism through which the SMAs can control action 64 execution. Indeed, although the power of the theta band over the motor system could reflect an 65 inhibitory mechanism, recent evidence suggests that the phasic period of frontal theta is crucially 66 related to performance in cognitive tasks that involve frontal cortical circuits (11). In particular, theta 67 band phase coherence within and across frontal, motor and parietal regions seems to correlate with a 68 higher accuracy in rule-based decision making $(12,13)$ and memory tasks $(14)$, as well as shorter 69 reaction times during attentional paradigms (15). Despite this growing body of evidence, a direct link 70 between theta oscillatory phase and the executive control of action is still missing (although see 71 (16)). In summary, it remains unclear whether the human SMAs are involved in behavioral switching, 72 whether they have an inhibitory or faciliatory role in action reprogramming and what is the neural 
73 mechanism is underlying this putative role. To address these questions, we tested the ability to

74 switch between automatic and deliberate control of action in three (pre-operation) epileptic subjects

75 with iEEG medial-frontal implants in the supplementary motor areas (BA6). Subjects performed a

76 variation of the Serial Reaction Time Task (SRTT) (17), a paradigm that requires the execution of a

77 sequence of repetitive visually-guided key-presses, which becomes progressively automated. In a

78 small subset of trials, the automatic sequence is unpredictably interrupted by the appearance of a cue

79 (switch trials), which required the subjects to halt the ongoing action sequence and press an

80 alternative un-cued key (switch trials).

81 Consistently with the animal literature $(4,6)$, we find that the SMAs are active during switch trials but 82 not during automatic ones. We also observe that the response time at the single trial level can be

83 predicted from the movement related cortical potential (MRCP) (18). Notably, the ability to detect

84 MRCP peaks at the single trial level allowed us to align the iEEG signals recorded from the SMAs to an

85 endogenous neural event predictive of behavior. This was crucial to minimize intra- and inter-subject

86 variability (19) and to unveil the intrinsic neural dynamics independent of an exogenous (i.e. cue

87 presentation) event-locked analysis $(12,20,21)$. We show that the single-trial theta band phase

88 coherence $(22,23)$, i.e. the contribution of a single trial to the overall phase consistency across trials,

89 were predictive of motor performance at the single trial level, with higher phase coherence

90 correlating with shorter reaction times. Neither amplitude nor power of the MRCP had such

91 predictive power. To further investigate the neurophysiological link between low-frequency

92 oscillations and behavior, we computed the cross-frequency coupling between theta phase and the

93 amplitude of higher frequency activity. This analysis revealed a significant increase of theta-gamma

94 phase-amplitude coupling associated with shorter response latencies suggesting a modulatory effect

95 of theta rhythms on local neuronal activity.

96 Altogether our results directly support the role of human SMAs in the control of action switching.

97 Moreover, they reveal a possible mechanism of cognitive control based on the entrainment of high-

98 frequency neuronal events by low-frequency oscillations in a cognitive control version of the nested

99 frequency theta-gamma code (24). This interpretation provides further support for the growing body

100 of evidence pointing to the role of phase synchrony and modulation in the theta band as a

101 fundamental operational mode of frontal executive function.

104 RESULTS

\section{Task and behavioral results}

108 In order to explore the neural dynamics underlying behavioral performance in deliberate action 109 switching, three human subjects implanted with intracranial electrodes (iEEG) in the supplementary 
110 motor areas (SMAs) (fig. 1-B), performed a variation of the serial reaction time task (17,25) (fig. 1-A).

111 The task was composed of two consecutive blocks for a total of 565 trials. The first block (trial 1-60)

112 required the participants to learn to perform a repetitive sequence of key-presses $(\mathrm{N}=5)$ on a touch-

113 screen keyboard by tapping on a visual cue (green, presented for $500 \mathrm{msec}$ or until pressed) until

114 they reached automaticity. Automaticity was defined as the decrease of inter-key-interval (IKI) to an

115 asymptotic value, indicating that the subject effectively internalized the motor sequence and did not

116 rely on visual feedback (17) (fig. 1-C). We observed a significant decrease in IKI between early and

117 late training trials (fig. 1-D) (t-test unpaired between trials 1-20 (N=20) and trials 40-60 (N=20):

118 SBJ1, t=5.30, $\mathrm{p}<10^{-05} ; \mathrm{SBJ} 2, \mathrm{t}=5.71, \mathrm{p}<10^{-05} ; \mathrm{SBJ} 3, \mathrm{t}=4.08, \mathrm{p}<10^{-03}$ ) and its variability (fig. 1-E) ( $\mathrm{t}$-test

119 unpaired: $\mathrm{SBJ} 1, \mathrm{t}=2.21, \mathrm{p}=0.04 ; \mathrm{SBJ} 2, \mathrm{t}=2.45, \mathrm{p}=0.02$; $\mathrm{SBJ} 3, \mathrm{t}=3.28, \mathrm{p}=0.004$ ), confirming that the

120 subjects performed the sequence in an automatic manner (26).

121 In the second block of the task (trial 61-565), subjects were required to perform the learned

122 sequence of visually guided key presses. However, these were interrupted by the unpredictable

123 appearance of a switch cue (red circle) at pseudo-random intervals $(7+-2 \mathrm{sec})$. When the switch cue

124 appeared, subjects had to interrupt their ongoing automatic motor sequence and press a specific

125 uncued key (fixed for each subject throughout the experiment) as instructed during the training

126 phase (switch trials). Subject 1 and 2 requested to interrupt the experiment prematurely. In total we

127 collected 50 switch trials for SBJ1, 57 for SBJ2 and 80 for SBJ3. Subjects were able to successfully

128 interrupt the ongoing action sequence for most of the switch trials. Switch trials where subjects

129 failed to interrupt the ongoing motor sequence and pressed the next key in the sequence (SBJ1, 14\%;

130 SBJ2, 20\%; SBJ3, 10\%) were excluded from the analysis. The behavioral analysis of the key-presses

131 showed consistently longer response in switch trials compared to automatic response trials (fig. 1-F)

132 (t-test unpaired: SBJ1, $\mathrm{t}=-39.59, \mathrm{p}<0.01 ; \mathrm{SBJ} 2, \mathrm{t}=-32.96, \mathrm{p}<0.01 ; \mathrm{SBJ} 3, \mathrm{t}=-71.56, \mathrm{p}<0.01$ ). This

133 increased latency in response time was accompanied by a greater variability ranging from about 600

134 to $1300 \mathrm{~ms}$ (fig. 1-G). Hence, the motor response dynamics in automatic trials were consistent and

135 stereotyped whereas in switch trials subjects were variably faster or slower than their mean

136 performance at each trial. This difference cannot be explained as a learning effect, as we did not find

137 a significant correlation between trial order and switch response latencies (Pearson's correlation

138 coefficient: SBJ1: $\mathrm{R}=-0.11, \mathrm{p}=0.18$; SBJ2: $\mathrm{R}=0.08, \mathrm{p}=0.37$; SBJ3: $\mathrm{R}=-0.16, \mathrm{p}=0.07$ ). In addition, it cannot

139 be consistently explained by the position of the switch cue within the sequence, which showed a

140 significant effect only in one subject (one way ANOVA: SBJ1: $F(2,40)=2.546, p=0.091$;

141 SBJ2: $F(2,43)=2.002, p=0.147 ; \operatorname{SBJ}: F(2,69)=4.685, p=0.012)$. This raises the question whether the

142 difference in performance in automatic and switch trials can be explained in terms of the properties

143 of the neuronal process underlying the control of action switching.

144 
a

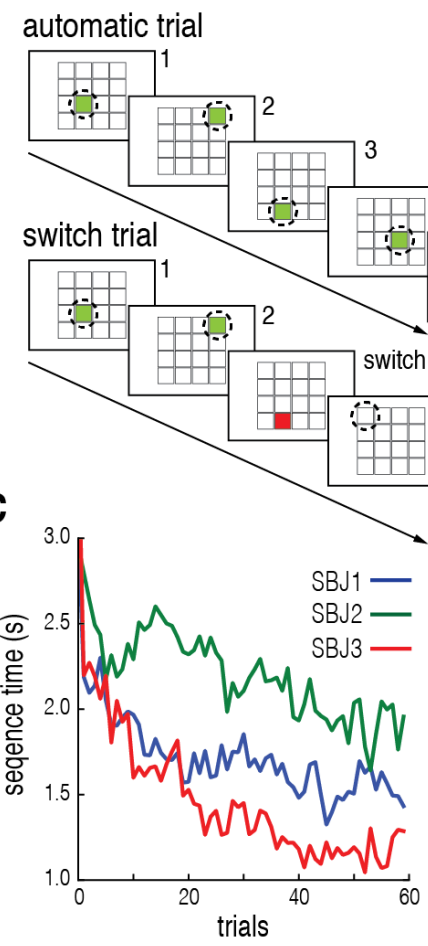

b

e

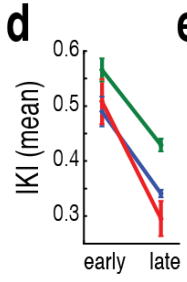

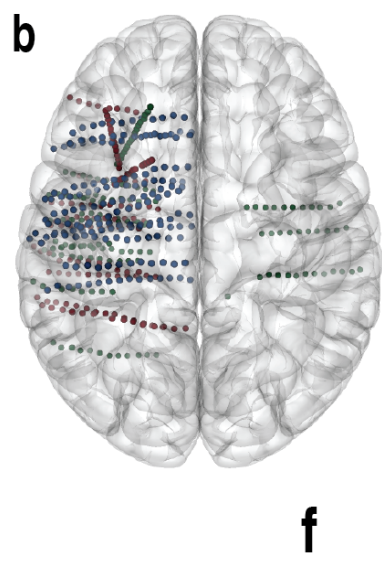

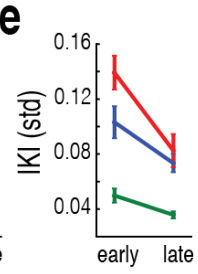

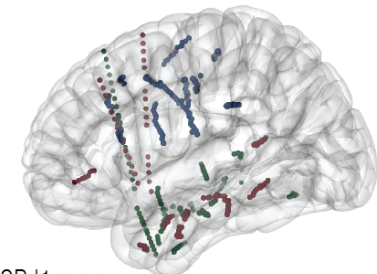

SBJ1 $\bullet \bullet$ $\mathrm{SBJ} 2 \bullet \bullet \bullet$ SBJ3 $\bullet \bullet \bullet$

g
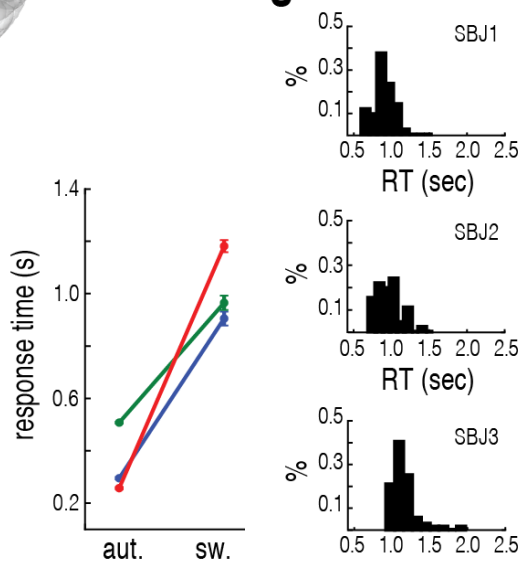
RT (sec)
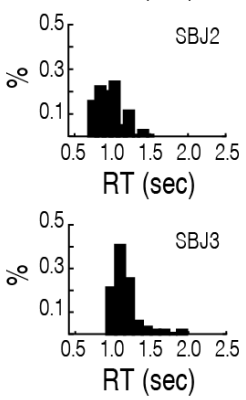

Figure 1. Experimental protocol, recording locations and behavioral performance. a. Serial reaction time

task. During automatic trials subjects are required to perform a series of visually guided key presses (green key)

following a fixed pseudo-randomly generated sequence. During switch trials subjects are presented with a switch cue (red key in a fixed position) appearing at a random step of the sequence, requiring them to interrupt the ongoing motor sequence and press a specific uncued key. b. Projection of the locations of relevant contact points in MNI coordinates for each subject. c. Evolution of trail duration during training per subject. d. Mean Inter-Key-Interval (IKI) in seconds during early (trial 1-20) and late (trial 40-60) phases of training for each subject. e. IKI standard deviation during early (trial 1-20) and late (trial 40-60) phases of training for each subject. f. Comparison between response time during automatic and switch trials for each subject. Switch responses are collected from all the valid trials in the switch condition from the appearance of the switch cue to the key press. Automatic responses comprise the same number of key presses sampled from the automatic sequence at random from the pool of automatic trials (SBJ 1: $\mathrm{N}=43$; SBJ 2: $\mathrm{N}=46$; $\mathrm{SBJ}$ 3: $\mathrm{N}=72$ ). g. Normalized distribution of response time during switch trials for subject. Response time computes as in $\mathrm{f}$.

164 It is believed that the SMAs contribute to cognitive control in particular the switching between 165 automatic and deliberate action (2-4). We validated this hypothesis by training a classifier to identify 166 the trial type (automatic vs. switch), by the signals obtained from all the available contact points for each individual subject (see Methods). This classification shows that the signals obtained from the 
168 medial-frontal cortex, and in particular the SMAs (BA6C), are most predictive of switch trials (F1

169 score=0.7) followed by the motor cortex (BA4) (F1 score $=0.57$ ) and the lateral premotor cortex

170 (B6L) (F1 score = 0.52). In particular, we observe that the amplitude of the iEEG local field potentials

171 (LFPs) measured in the SMAs is predictive of the type of trial (automatic or switch) (fig. 2-A, B).

172 Following this step, we restricted our analysis to the relevant contact points in the SMAs in order to

173 determine the precise role of SMAs in switching from automatic to deliberate behaviors.

174 Analyzing the time evolution of the SMAs iEEG we observe that during switch trials, a significant

175 movement related cortical potential (MRCP) preceded the response in all three subjects. In contrast,

176 this MRCP was not present during automatic trials (T-statistics cluster permutation $(\mathrm{N}=1000)$

177 analysis: SBJ1, t=34.09, $\mathrm{p}<10^{-4} ; \mathrm{SBJ} 2, \mathrm{t}=21.20, \mathrm{p}<10^{-4} ; \mathrm{SBJ} 3, \mathrm{t}=29.69, \mathrm{p}<10^{-4}$ ) (fig. 2-B). To control

178 whether this MRCP encoded the motor sequence initiation rather than a switch action per se (27), we

179 aligned the LFPs for each subject to the cue indicating the beginning of the automatic motor sequence

180 (not shown). We found no significant increase in amplitude confirming that the earlier observed

181 MRCP was specific to automatic-deliberate switching (T-statistics cluster permutation $(\mathrm{N}=1000)$

182 analysis: $S B J 1, t=7.64, p=n . s . ; S B J 2, t=4.73$, $p=n . s . ; S B J 3, t=8.13, p=n . s$.$) .$

183 Our results confirm that the SMAs mediate the execution of actions that require cognitive control,

184 such as switch trials and are not involved in the control of over-trained sequential motor responses

185 nor the initiation of automatic sequences (1).

186 Due to the high resolution of intracranial EEG, we aimed to identify robust MRCPs at the single trial

187 level in order to determine what features of the neural signal were predictive of behavioral

188 performance. To achieve this, we extracted the temporal and amplitude information for each event

189 by band-pass filtering the signal at low frequencies (1-2 Hz) and further extracting the absolute peak

190 (fig. 2-C-1,2) (18,28). We further analyzed the time window from the switch cue presentation to the

191 MRCP peak (TP), the time window from the peak to the key press (FP) and the peak amplitude (A) in

192 relation to the response time at each trial. Temporal analysis of the MRCP showed a marked

193 correlation between its time to peak (TP) and reaction time with respect to cue presentation at the

194 single trial level (Pearson's correlation coefficient: SBJ1, $R=0.481, p<0.001$; SBJ2, $R=0.779, p<10^{-10}$,

$195 \mathrm{SBJ} 3, \mathrm{R}=0.499$, $\mathrm{p}<10^{-5}$ ) (fig. 2-E). A similar positive correlation was found between single trial time-

196 from-peak to key-press (FP) and the response time (Pearson's correlation coefficient: SBJ1, R=0.698,

$197 \mathrm{p}<10^{-7} ; \mathrm{SBJ} 2, \mathrm{R}=0.707, \mathrm{p}<10^{-8}, \mathrm{SBJ} 3, \mathrm{R}=0.464, \mathrm{p}<10^{-5}$ ) (fig. 2-F). In contrast, peak amplitude showed

198 no consistent effect on response times and only in one subject reached statistical significance

199 (Pearson's correlation coefficient: SBJ1, R=0.141, p=0.371; SBJ2, R=0.314, p=0.035, SBJ3, R=0.06,

200 p=0.959) (fig. 2-G). To further quantify the relevance of the time to peak and amplitude aspects of the

201 MRPC for switch behavior we fit a linear model for each variable and computed the explained

202 variance $\left(\mathrm{R}^{2}\right)$ with respect to

203 
a

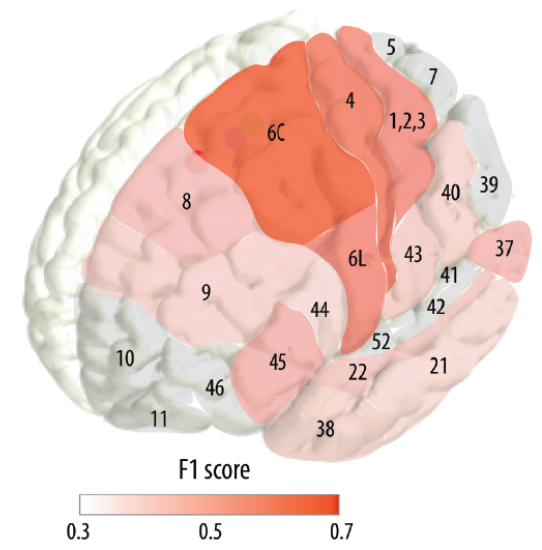

b

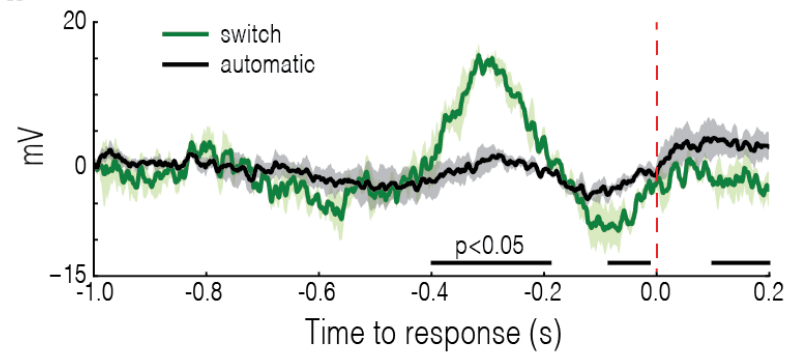

C

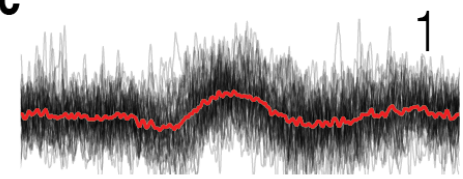

d
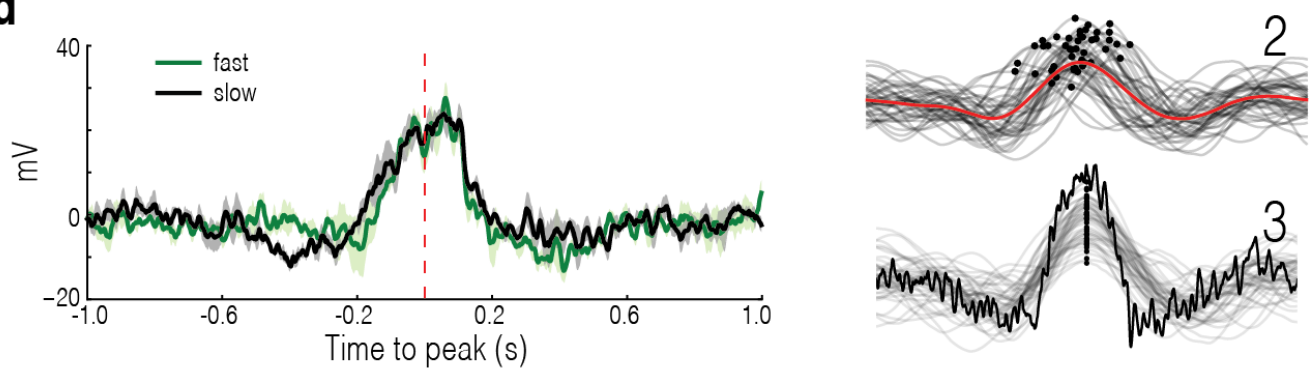

e

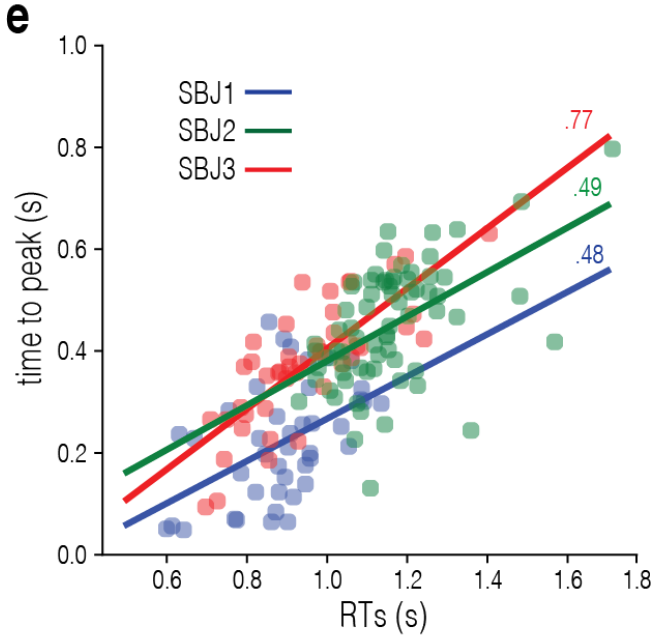

f

g
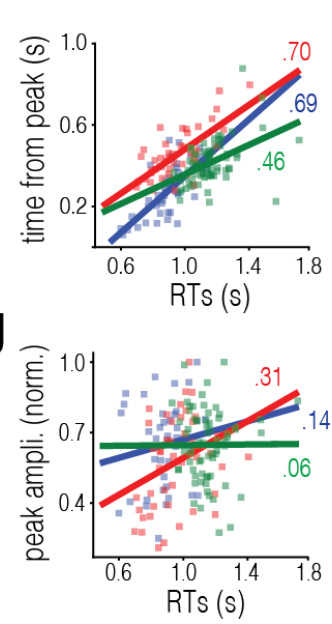

$\mathrm{h}$

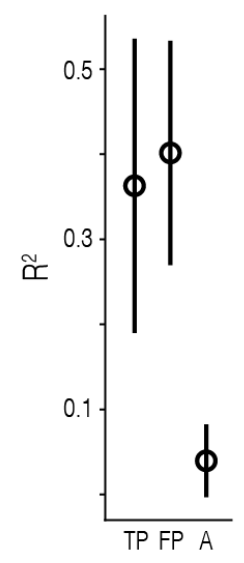

Figure 2. Neural response in SMAs. a. Classifier mean prediction accuracy of switch trials projected over a Brodmann atlas. Red hue represents the classification accuracy (F1 score) associated with the areas that were recorded, gray denotes areas not present in the recording. b. Motor Related Cortical Potential (MRCP) in SMAs during switch (green) and automatic (black) trials aligned to motor response (red dashed). Switch trials are aligned to the key-press in response to the switch cue (key press of the uncued key), automatic trials are aligned to the last key-press of the automatic sequence. Mean and SEM for each subject. Bar indicates the intersection of 
212 significant temporal windows for all the subjects $(\mathrm{p}<0.05)$. c. Example of alignment of single trial MRCPs to the

213 relative peak for one subject. 1. Key-press aligned MRCPs for each switch trial (black) and mean (red). 2. 2Hz

214 Low passed MRCPs for each trial (black) and mean (red). Black dots indicate the detected peaks. 3. Alignment of

215 low passed filtered MRCPs by their relative peak and unfiltered average. d. MRCP in SMAs during fast and slow

216 responses during switch trials (trials sorted by the median of the response time distribution) aligned to the peak

217 of the band-passed MRCP (red dashed). Mean and SEM of subjects. e. Relationship between time of response and

218 of cue presentation to MRCP peak during switch trials for each subject. Solid line indicates the linear fit of the

219 data and Pearson's correlation coefficient. f. Relationship between response time and time from MRCP peak to

220 key-press during switch trials for each subject. g. Relationship between response time and MRCP peak

221 amplitude during switch trials for each subject. h. Mean $\mathrm{R}^{2}$ linear regression coefficient between response time

222 and time from cue to peak (TP), time from peak to key-press (FP) and peak amplitude (A). Each score is obtained

223 on the test set as a result of an independent regression for each regressor trained on $70 \%$ of the available

224 aggregated trials and tested on the remaining 30\%. Mean scores and standard deviation are the result of a 100-

225 fold cross-validation.

226

the response time (fig. 2-H). This analysis confirmed that the temporal dynamics of the MRPC in terms of time-to-peak from cue onset and time-from-peak to response could accurately predict performance while excluding amplitude as a reliable predictor of motor behavior.

230 We subsequently analyzed whether the profile of the MRPCs showed any behavior dependent 231 modulation by comparing fast and slow switch trials (fig. 2-D). For each subject, trials were aligned to 232 the time of the peak of the band-passed MRCP (as shown in fig. 2-C-3). Further, these trials were 233 sorted into two groups according to the median value of the response time distribution (i.e. fast and

234 slow) in order to obtain an equal number of trials for each group and potential differences between 235 groups tested using cluster-based permutation analysis. This analysis revealed no differences 236 between fast and slow switch trials (T-statistics cluster permutation $(\mathrm{N}=1000)$ analysis: $\mathrm{SBJ} 1, \mathrm{t}=5.87$, $237 \mathrm{p}>0.05 ; \mathrm{SBJ} 2, \mathrm{t}=9.23, \mathrm{p}>0.05 ; \mathrm{SBJ} 3, \mathrm{t}=8.61, \mathrm{p}>0.05$ ) suggesting that, although the MRPC is a 238 characteristic neural signature of switch actions, it does not encode information predictive of 239 behavioral parameters such as response time.

240 Altogether, these results suggest that the SMAs are recruited for deliberate control of switching 241 behavior and that the temporal features of the MRPC, i.e. phase, are decisive in controlling the 242 response time (29).

245 Theta phase aligns in faster actions

247 We hypothesized that oscillatory dynamics in the theta range could constitute a neural signature of 248 cognitive control by mediating deliberate action switching. Previous reports have suggested a strong 
a

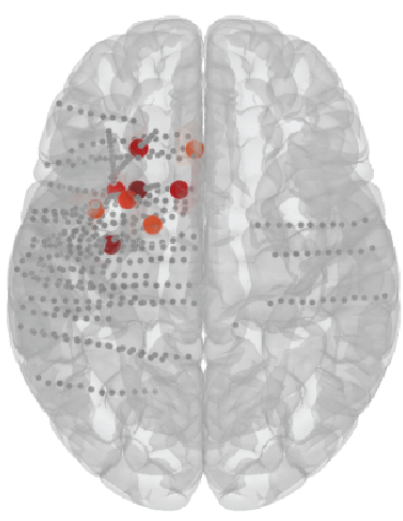

C

ITPC fast-slow difference

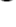

b
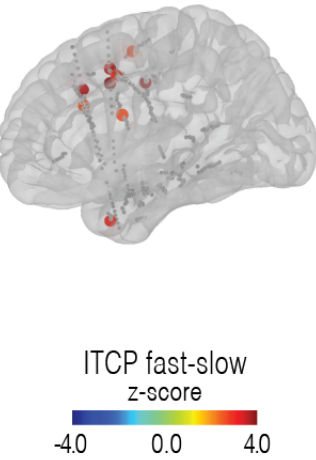
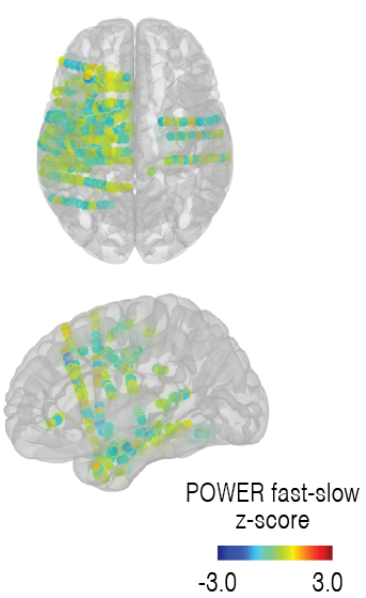

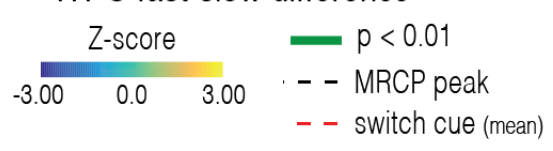

\section{STPC-RT correlation}
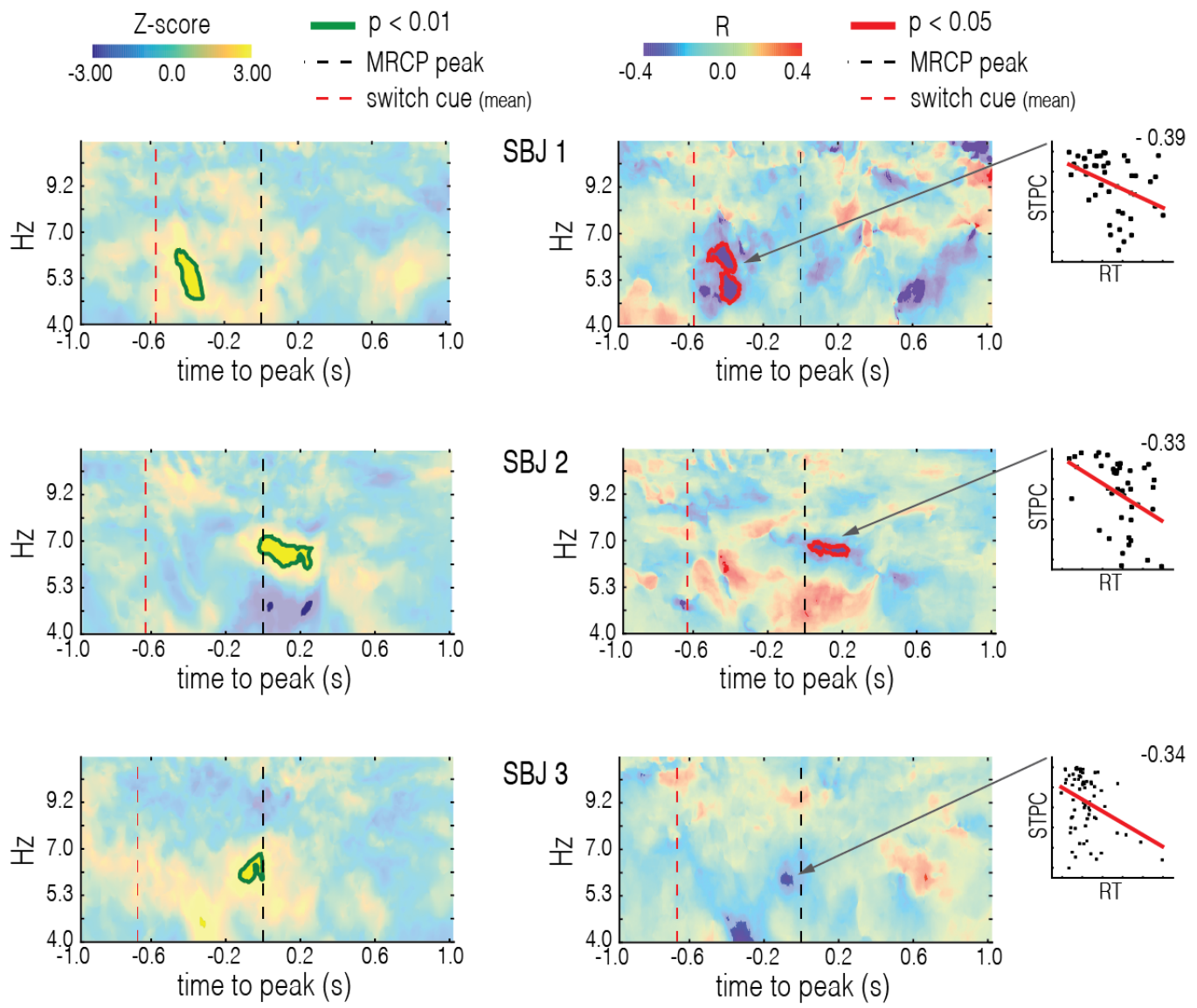

253 Figure 3. Phase alignment and performance. a. Inter-trial phase coherence difference (z-score) between fast 254 and slow (fast-slow) switch trials for the combination of the significant contact points for each subject projected 255 on the MNI space. Contact points showing a significant cluster of time-frequency bins $(\mathrm{z}>2.58$, Montecarlo $\mathrm{p}$ 256 value $<0.05$ ) in the time window $-1 \mathrm{~s}$ to $1 \mathrm{~s}$ centered to the peak of the MRCP are represented in color. b. Power 
difference (z-score) between fast and slow (fast-slow) switch trials for the combination of all the contact points available from all the subjects projected on the MNI space. No contact point reached a statistically significant level, therefore no masking is applied to this figure. c. Single subject statistical analysis. left. ITPC normalized difference between fast and slow (fast-slow) switch trials. Trials are aligned by the relative peak of the MRCP (black dashed line). Red dashed line indicates the average cue onset time with respect to MRCP peak. Green outline indicates region with Monte Carlo $\mathrm{P}$ value $<0.01$. right. Spearman's R correlation coefficient between Single Trial Phase Coherence (STPC) and Response Time (RT) computed for each time frequency bin during switch trials. Trials are aligned by the relative peak of the MRCP (black dashed line). Red dashed line indicates the average cue onset time with respect to the MRCP peak. Red profile indicates Monte Carlo P value $<0.05$. box. Relationship between Response Time and STPC for the significant area shown in the time-frequency plot shown to the left. Red line indicates linear fit.

implication of phase dynamics in cognitive control $(11,12,16)$ suggesting the possibility that stereotypical phase profiles could underlie deliberate action modulation. To detect potential stereotypical phase patterns underlying the differences in response times (RT), we first aligned the LFPs to the peak of the MRCP. Note that this is not only a necessary step to filter out inter- and intra- subject differences so as to compare phase profiles across conditions (19), but it could also reveal oscillatory patterns that are removed when using exogenous events as reference $(8,12,20)$. Further, we sorted the switch trials for each subject into two classes of equal size fast and slow), by splitting the RT distributions by their median value. For each subject and each available contact point, we then calculated ITPC for fast and slow trials and computed the normalized phase coherence difference following the method described in (30) (see Methods). Class comparison revealed a significantly larger phase alignment in fast trials compared to slow trials localized in the SMAs for all the subjects. To verify the specificity of this result we applied Z-statistics cluster permutation analysis to all the available contact points in order to identify significant differences in ITPC between fast and slow trials (see Methods) and further projected in MNI space the contact points with a significant difference $(\mathrm{p}<0.05)$ in the time window between -0.6 and $0.4 \mathrm{~s}$ with respect to the peak of the MRCP (Figure 3-A, see Methods). We observe that, contact points with significantly stronger phase alignment fall in between Brodmann area 6 and 9 for subjects 1 and in Brodmann area 6 for subjects 2 and 3. This effect was found in the theta frequency range between 4 and $8 \mathrm{~Hz}$ (fig. 3-C left for an example for each subject) with an onset varying across subjects between 0.5 and 0.1 seconds before the MRCP peak, possibly due to implant location differences (i.e. more frontal for subject 1). Similar theta range variability was detected in the contact points of individual subjects in areas anatomically or functionally related to the SMA. In particular, in the anterior cingulate cortex (ACC) of subject 3 and in the temporal lobe of subject 2 (see Table 1 for a list of the contact points with significant differences $(p<0.05)$ in phase alignment between fast and slow trials). 
294 To further confirm the role of theta phase dynamics with respect to action switching, we applied a 295 single-trial analysis to a subset of contact points in the SMAs that showed the reaction speed and 296 phase alignment effect. Our specific goal was to determine the contribution of inter-trial phase 297 coherence to motor performance. In particular, we computed the total inter-trial phase coherence 298 (ITPC) for each time-frequency bin and inferred the individual contribution of intra-trial coherence 299 by computing a Single Trial Phase Coherence (STPC) pseudo value following $(22,23)$. Finally, for each 300 time-frequency bin, we computed a Spearman's R correlation between the trial response times (RT) 301 and the relative STPCs (see Methods). We observe a negative correlation between RT and STPC in the $3024-8 \mathrm{~Hz}$ frequency band once aligned to the peak of the MRCP (fig. 3C, right) (Z-statistics cluster 303 permutation ( $\mathrm{N}=1000)$ analysis: $\mathrm{SBJ} 1, \mathrm{z}>1.96, \mathrm{p}<0.001$; $\mathrm{SBJ} 2, \mathrm{z}>1.96, \mathrm{p}<0.001 ; \mathrm{SBJ} 3, \mathrm{z}>1.96, \mathrm{p}=0.07)$. 304 Altogether these analyses reveal a stereotypic phase profile of theta oscillations locked to the motor 305 potential controlling action switching whose magnitude is monotonically related to the time of the 306 behavioral response at the single trial level.

307 Importantly, it has been argued that phase coherence may be induced by increases in the power of 308 the oscillations, thus constituting an evoked rather than an actual phase alignment (29). We 309 controlled for this possibility by calculating the difference in power between fast and slow trials and 310 compared the obtained z-scored differences with those obtained in the ITCP domain (fig. 3-B). This 311 analysis confirms a significant effect of ITPC on response time in the absence of significant 312 differences in power suggesting that the detected phase alignment is likely the result of an actual 313 phase coding mechanism within the theta range.

314 Altogether, these results suggest that the SMAs play a central role in controlling the ability to switch 315 from the execution of an automatic motor sequence to the execution of a deliberate action prompted 316 by an unexpected cue. The enhanced synchronization of theta frequencies in faster switch trials, in 317 absence of power differences, supports the role of phase dynamics as a mediating mechanism that 318 facilitates the execution of deliberate movements. In addition, the differences found in the ACC and 319 temporal lobe support the existence of a functional network involved in switching. Within this 320 network the ACC could be responsible for selectively biasing processes in favor of task relevant 321 information during high conflict switch trials (31). The temporal lobe in turn might encode for 322 arousal or surprise elicited by the unexpected cue $(32,33)$ or support the mnemonic aspects of the 323 decision making process (e.g. retrieving what key should be pressed once the cue is presented) (34).

\begin{tabular}{|l|l|l|l|l|}
\hline Subject & $\begin{array}{l}\text { Contact } \\
\text { point }\end{array}$ & MNI coordinates & $\begin{array}{l}\text { Brodmann } \\
\text { Area }\end{array}$ & ITPC statistics \\
\hline 1 & I'12 & $-23,713 ; 25,731 ; 35,165$ & BA9 & Z=3.547, MC_p=0.004 \\
\hline 2 & T'3 & $-32,098 ; 10,264 ;-38,737$ & BA36 & Z=3.210, MC_p=0.022 \\
\hline 2 & X'10 & $-24,089 ; 10,467 ; 38,760$ & BA6 & Z=4.003, MC_p=0.014 \\
\hline
\end{tabular}


325

\begin{tabular}{|l|l|l|l|l|}
\hline 3 & K'2 & $-8,865 ; 10,540 ; 47,061$ & BA32 & Z=3.546, MC_p=0.016 \\
\hline 3 & O'10 & $-39,712 ; 3.550 ; 30,963$ & BA48 & Z=2.841, MC_p=0.026 \\
\hline 3 & M'9 & $-33,840 ;-9,556 ; 39,289$ & BA6 & Z=3.737, MC_p=0.022 \\
\hline 3 & R'4 & $-18,703 ;-2,314 ; 56,739$ & BA6 & Z=2.836, MC_p=0.04 \\
\hline 3 & K'7 & $-27,784 ; 6,911 ; 42,980$ & BA6 & Z=3.005, MC_p=0.046 \\
\hline 3 & B'1 & $-3,148 ; 25,277 ; 26,200$ & BA32 & Z=2.727, MC_p=0.042 \\
\hline
\end{tabular}

Table 1. List of all the contact points where the difference in ITCP between fast and slow trials reached statistical significance.

\section{Cross-frequency coupling predicts faster movements}

We have shown that the speed of deliberate action switching is accompanied by theta phase alignment. However, the direct physiological link needed to support the hypothesis that theta phase dynamics modulate performance via phase-dependent neural activity needs to be established. We sought to answer this question by determining the modulatory effect of theta phase on local highfrequency activity with the hypothesis that higher modulation could support faster switch actions (fig. 4-A), an analysis for which a measure of cross-frequency Phase-Amplitude Coupling (PAC) is particularly suited $(35,36)$.

We restricted our analysis to the contact points and the temporal windows of approximately $400 \mathrm{~ms}$ where a significant increase in phase alignment was detected (Figure 3C). To achieve the temporal resolution necessary for this type of analysis we obtained one surrogate signal for fast and slow trials for each subject by concatenating the respective single trial windows for all the SMAs contact points for each subject (see Table 1 for a list of contact points). Further, we computed PAC values (using the GLM approach (37)) between 4-8 Hz phase (modulatory frequency) and the amplitude of higher frequencies (10-100 Hz in steps of $2 \mathrm{~Hz}$, modulated frequency), and obtained the difference between the two types of trials. Further, to test the significance of the difference between fast and slow trials, we computed the expected difference in PAC under the null hypothesis by shuffling the two types of trials and extracted the $\mathrm{z}$ threshold corresponding to $\mathrm{p}=0.01$ (38) (see Methods). We observed an increased modulatory effect of the theta oscillatory phase on the amplitude of frequency bands in the low gamma range $(30-80 \mathrm{~Hz})$, which was consistent across subjects (Z-statistics permutation ( $\mathrm{N}=1000$ ) analysis: $\mathrm{SBJ} 1, \mathrm{z}>2.58, \mathrm{p}=0.027$; $\mathrm{SBJ} 2, \mathrm{z}>2.58, \mathrm{p}=0.034 ; \mathrm{SBJ} 3, \mathrm{z}>2.58, \mathrm{p}=0.029$, fig. 4-B,D). In addition, a significant modulatory effect was found in the beta range (i.e. $30 \mathrm{~Hz}$ ) for one subject (SBJ1, $\mathrm{z}>2.58, \mathrm{p}=0.019$ ). Further analysis revealed no difference in absolute gamma amplitude between trial 
354 types (SBJ1, p=0.23; SBJ2, p=0.26; SBJ3, p=0.65), suggesting that the magnitude of phase modulation, 355 rather than activity per-se, has a direct effect on controlling performance (fig. 4-D). This result 356 supports a neurophysiological link between theta phase coherence and deliberate control of the 357 action switching through the modulation of high-frequency activity, often interpreted as a correlate 358 of local population activity (35).

a

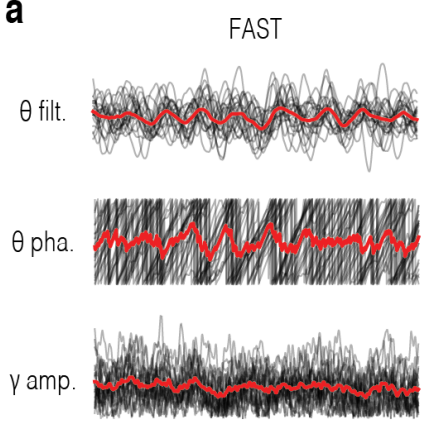

SLOW
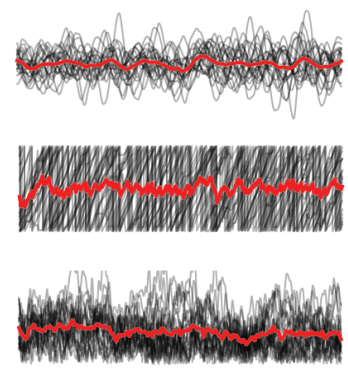

b

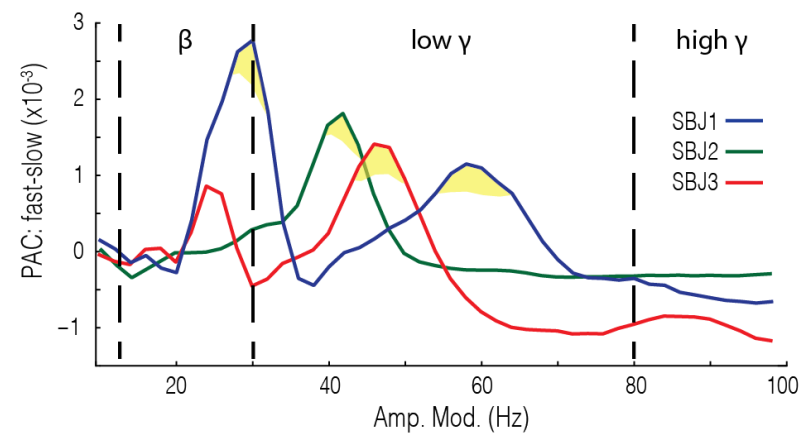

C

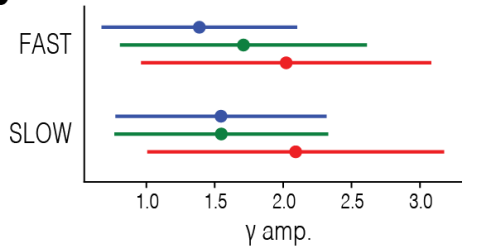

d
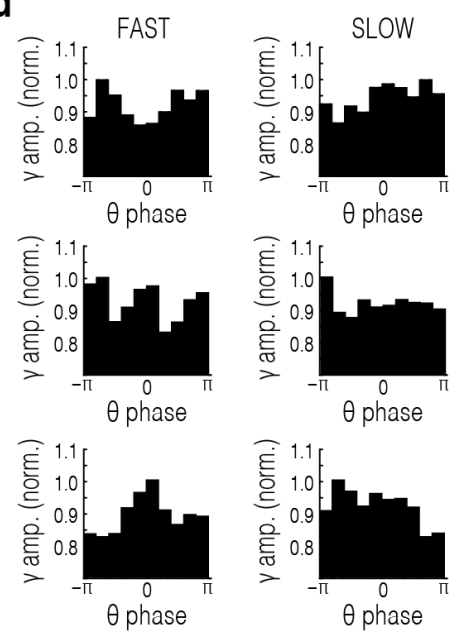

Figure 4. Theta-gamma cross frequency coupling and its relation to performance. a. Example of oscillatory activity in SMAs during fast trials (left) and slow trials (right) for SBJ 2. Top. LFP traces of individual switch trials (black) and mean (red) filtered in the theta range (4-8 Hz). Center. Oscillatory phase of the individual LFP traces (black) and mean (red) shown in the above panel. Bottom. Amplitude of the individual LFP traces (black) and mean (red) filtered in the low gamma range (30-80 Hz). All traces are aligned to the peak of the MRCP and sorted by fast (<900 msec) and slow (>900 msec) response time. b. Difference in phase amplitude coupling (PAC) between fast and slow trials (fast-slow) for each subject comparing the phase of theta oscillations (4-8 Hz) to the amplitude of higher frequency bands $(\mathrm{x}, 30 \mathrm{~Hz}, 30-80 \mathrm{~Hz}$, and $80-100 \mathrm{~Hz}$ ). Yellow area indicates $p<0.05$. c. Quantification of mean gamma amplitude in fast and slow trials for each subject selected from statistically significant regions marked in yellow in panel b. Bar indicates standard error of the mean. $\mathbf{d}$. Distribution of gamma band amplitude (selected from statistically significant regions in b) over the phase of theta for fast (left) and slow (right) trials for each subject. 
377 Switching from automatic to deliberate behavior in response to environmental cues is a key aspect of 378 adaptive behavior, however, the substrate and dynamics supporting this ability in the human brain 379 are not fully understood. Here, we have addressed this question by analyzing the iEEG of the 380 neocortex subjects engaged in a switch task. We designed a variation of the Serial Reaction Time 381 Task (SRTT) where automaticity is detected as a progressive shift towards faster and more 382 stereotyped movements, indicating that sequences are performed on the basis of an internal habitual 383 representation and allowed to execute autonomously $(7,39,40)$. Using a classifier of the time-domain 384 features of the iEEG we localized a predictive signal of switch trials to the SMAs which confirmed its 385 role in action control. We found that by aligning trials to action initiation, stereotypical phase profiles 386 in the theta range emerged in the SMAs that predicted switch performance at the single trial level. In 387 addition, we showed that theta rhythms modulated high-frequency power in a performance388 dependent manner.

389 In our design, however, we extended the SRTT by introducing a switch cue the required the subjects

390 to interrupt the automated sequence and execute an alternative key-press. This alternative action 391 was un-cued, and it occurred at a low and variable rate, at unexpected positions in the sequence, in 392 order to both avoid habituation and maintain active deliberation. This manipulation is analogous to 393 the switch cue used in (6). However, in our setup, the alternative action was un-cued, to guarantee a 394 minimum amount of deliberation since subjects had to retrieve and implement a specific instruction 395 set from memory (5). During the switch trials, we found significantly longer response times, 396 suggesting a switch from automatic to controlled, deliberate processes $(3,6,41)$. Within the 397 psychological literature, this behavioral marker is known as switch cost (42), interpreted as the 398 result of an "act of control" that involves attentional, mnemonic and motor processes.

399 We found that activity in the SMAs could successfully predict whether the action was performed 400 deliberately or automatically, supporting the involvement of human SMAs in executive control. This 401 result extends previous evidence from primate studies that demonstrated a lateralized increase in 402 the firing rate of supplementary eye field neurons that exclusively accounted for successful 403 performance during switch but not automatic actions (4). Although the activity profile found in our 404 study is qualitatively similar to the one reported in (6), it remains unclear what the 405 neurophysiological link between low-frequency LFP events and firing rate is. One possibility is that 406 the motor potential we reported (fig. 2-b) is the result of the sum of synchronized 407 afterhyperpolarization events of SMAs neurons contributing to the extracellular field potential (43). 408 This pattern of activity is also consistent with human fMRI studies that reported a consistent 409 activation of the SMAs and the more anterior pre-SMAs during action switching $(1,41,44)$. For 410 example, SMAs are transiently active, together with the cingulate cortex, during the execution of 411 switch actions triggered by response conflict $(1,45)$. Besides, TMS induced inhibition of medial 412 frontal areas impaired the ability to switch between motor responses but not the ability to initiate 
413 action per se, an observation consistent with our results showing no significant change of activity

414 during sequence initiation (46). Moreover, contrary to previous reports (27), we excluded the

415 possibility that the neural signature of switching we report could encode sequence production, as no

416 SMAs activity was found during automatic trials. Instead, we demonstrate, to the best of our

417 knowledge, for the first time the movement-cortical potential (MRCP) as the prominent signature of

418 deliberate action switching in the human SMAs using iEEG. MRCPs are low-frequency potentials

419 generated in association with the planning and execution of a cued or self-paced voluntary

420 movement in premotor and motor regions (18). MRCPs can be decomposed in three distinct

421 components: a slow rise component, known as Readiness Potential, which is more prominent in self-

422 paced movements $(47,48)$. A fast decay component following the RP, called negative slope (NS),

423 which peaks at the moment of maximum negativity in concomitance of action initiation (motor

424 potential - MP). Finally, a rebound activity following movement initiation is associated with

425 movement monitoring. In our task, we identified a strong NS component preceding movement

426 initiation but no clear RP, possibly because the switch action was not internally (i.e. voluntarily)

427 generated. Previous literature indeed shows more prominent NS and absence of RP in cue-guided

428 movements compared to self-paced ones (49).

429 The absence of RP could also support a recent hypothesis by Schurger and colleagues (50). They

430 suggest that this apparent signature of volitional control could be attributed to an artefact arising

431 from the averaging of stochastic fluctuations in the neural activity over trials. Indeed, within the EEG

432 literature MRCPs are typically extracted from averaging across a large number of trials time-locked

433 to action initiation, in order to filter out the noise and account for artefacts introduced by scalp

434 diffusion (51). This procedure, however, generates an artificial build-up in the averaged signal that

435 reflects the integration of noise in a stochastic decision process (SDP) following a drift-diffusion

436 model, where neural activity reaches a "decision threshold" and drives action initiation due to the

437 combination of evidence and stochastic fluctuations. The latter is seen as the source of the variability

438 in response times (52). In our study, the higher resolution and low SNR of iEEG allowed to robustly

439 detect MRCPs at the single trial level directly from the neural tissue and to extract reliable MRCPs

440 features. This allowed for a single-trial analysis precluded in standard EEG analyses usually deployed

441 in the investigation of $\operatorname{RP}(8,20,21)$ that support the SDP hypothesis. From a time-domain

442 perspective, the key finding in the single trial analysis of switch trials is that the temporal aspects of

443 the MRCP peak, but not its amplitude, could accurately predict switch response times. This is at odds

444 with previous EEG reports that showed a significant difference in the amplitude of cortical potentials

445 depending on the speed and strength of the movement $(53,54)$. The difference between our and

446 previous results could be due to the different methods employed in the recording. LFPs indeed

447 represent the extracellular activity of neural populations, and they closely track responses in a

448 restricted area of the neural tissue. EEG, in turn, captures a more spatiotemporally extended yet 
449 attenuated LFP that integrates several square millimeters of superficial cortical activity and it may 450 reflect more diffuse macroscopic effects (43).

451 Another methodological advantage of detecting single-trial time domain features is the possibility to 452 align individual trials to the dynamics of endogenous events. This is a necessary step to compare 453 oscillatory patterns across different conditions and subjects, as it has been shown that phase 454 synchrony measures are sensitive to inter-subject and intra-subject temporal variability (19). In 455 addition, previous studies have shown that locking to endogenous events rather than to exogenous 456 ones (i.e. stimulus presentation) can reveal important and otherwise hidden dynamics (12). By 457 aligning individual trials to the MRCP peak, we found that phase synchrony in the theta band across 458 trials predicted switching actions, where higher synchrony is associated with faster trials. This result 459 suggests that action initiation is locked to stereotypical phase profiles in the theta range, suggesting a 460 mechanism by which neurons in the medial frontal cortex synchronize to convey cognitive control 461 signals to downstream motor areas. This also defines a contrast between our results and the SDP 462 hypothesis, by virtue of analyzing signals relative to an internal reference, i.e. MRCP, we reveal a 463 systematic phase code orchestrating action control that will appear stochastic when referenced to 464 external or overt events such as stimuli or responses.

465 Importantly, we have shown that high synchronicity is prominently and consistently observed in the 466 SMAs but significant differences were also observed in the anterior cingulate cortex and the temporal 467 lobe of individual subjects (fig. 2a). This result suggests that the SMAs do not operate in isolation 468 during action switching, but form part of a broader functional network that involves sensory and 469 mnemonic processes. Indeed it has been suggested that the dorsal part of the ACC could play a major 470 role in conflict resolution by facilitating the selection of task relevant information during high conflict 471 decisions $(31,55)$. The consistency between the activity in the ACC and SMAs we observe (fig. 3-a) 472 supports this hypothesis and the existence of a functional connection between the two areas $(55,56)$. 473 In addition, it has been recently shown that the medial frontal cortex can flexibly recruit the medial 474 temporal lobe during decision-making by means of phase synchrony in the theta band (34). This 475 dynamic functional link between mnemonic and motor processes could explain the higher ITPC in 476 the temporal lobe found in our data (fig. 3-A) and suggests the involvement of this area in supporting 477 the mnemonic aspects related to the task (e.g. to recall what action should be performed when an 478 unexpected cue is presented).

479 More generally, phase coding has been acknowledged in the human hippocampus and temporal lobe 480 in mnemonic processes. In particular, (57) found that oscillations in the theta band reset their cycle 481 after stimulus presentation, leading to strong patterns of inter-trial phase coherence that correlate 482 with memory encoding. Similar phase resets in the theta range are found in frontal circuits with 483 greater synchrony underlying correct trials (58) and are thought to mediate attentional shifts. The 484 temporal patterns found in our study could, therefore, be the result of a switch cue induced phase 485 reset, encoding action related information, where the magnitude of the reset and its entrainment of 
486 local activity drives faster motor responses. The cycle of theta oscillations could indeed carry 487 patterns of information by modulating the amplitude of higher-frequencies in the gamma range (24).

488 Theta-gamma code is believed to represent a fundamental information-processing mode of the brain 489 responsible for sequential item encoding long-term and working memory within memory areas (59).

490 Recently, signatures of the theta-gamma code have been found in frontal cortical circuits during 491 cognitive control tasks. (12) for example, found increased theta-gamma phase-amplitude coupling 492 between prefrontal and motor regions when decisions followed higher order rules. Few reports have 493 linked phase coding to single trial behavioral performance, as in (15) where the strength of phase494 amplitude coupling in frontal and parietal regions correlated with reaction times during the 495 allocation of visuospatial attention. Similarly, we report distinct theta-gamma coupling modulated by 496 behavioral performance which suggests that theta range activity reflects a cognitive control signal 497 that entrains the gamma frequency activity of local neuronal populations (35).

498 Altogether these results contribute to a growing body of literature that identifies frontal theta phase 499 coding as a mechanism promoting cognitive control of behavior (11). Low-frequency oscillations 500 indeed could be a means of information exchange across distant populations within the same 501 functional network $(13,35)$. For example, (16) found fronto-parietal theta synchrony in primate in 502 primate to be predictive of the ability to correctly switch from automatic to deliberate control. 503 However, differently from our analysis, they did not find a relationship with the response time. A 504 similar synchronization pattern was found between the medial frontal cortex and the basal ganglia 505 (60).

506 Low-frequency oscillatory patterns could also be the result of cortical travelling waves in the theta 507 and alpha bands that have been identified as a mechanism through which information propagates 508 throughout cortical networks (61). Importantly, the spatial and temporal consistency of travelling 509 waves in the prefrontal cortex has been shown to have a facilitatory effect on working memory 510 retrieval, with greater synchrony predicting faster responses (21). Our results suggest a similar effect 511 within supplementary-motor circuits with phase synchrony having a facilitatory effect on deliberate 512 behavior. Contrary to this hypothesis, it has been suggested that medial-frontal theta oscillations may 513 also reflect inhibitory control by mediating action-slowing during situations of conflict and error (9). 514 In particular, human EEG studies testing interference tasks reported increased power in the theta 515 range during high-conflict trials correlating with an increase in response time, both prospectively 516 and retrospectively (i.e. post-error slowing) (8-10). Even though we did not explicitly test for this 517 aspect within our paradigm, we cannot confirm the role of theta as a signature of inhibition for three 518 reasons. Firstly, our analysis did not reveal any distinctive role of theta oscillatory power. In addition, 519 theta oscillatory phase alignment was found to reduce rather than increasing the response time on 520 deliberate switch trials. Finally, in our experiment, the detected pattern of synchronized activity 521 emerged by aligning the LFP of each trial to the peak of an action related event. If theta 522 synchronization represented an inhibitory signal, this event would not be strictly dependent on 
523 action execution and it would be expected to rise consistently earlier than the peak of the MRCP (62).

524 This discrepancy might again be due to the differences in resolution and specificity of iEEG versus

525 EEG recordings.

526 Overall, our results suggest that within frontal cortical networks, theta oscillations encode a control

527 signal that promotes the execution of deliberate actions. In particular, we propose that the power

528 and phase of theta oscillations may reflect different functional roles, where the former locally

529 encodes a general conflict signal and the latter serves as a long-range communication channel

530 facilitating cognitive control (63). This proposal is in agreement with recent accounts of cognitive

531 control that essentially see action selection (i.e. facilitation) and inhibition as two faces of the same

532 coin and therefore postulate complementary neural mechanism underlying these functions $(64,65)$

533 such as the power and phase of theta band oscillations. This further confirms that phase coding is a

534 fundamental representational format deployed by the brain.

535

536

537 METHODS

538

\section{Data collection}

540

541 Data were collected from three right-handed subjects with intractable epilepsy, temporarily

542 implanted with intracranial electrodes (iEEG) as a part of a pre-operation procedure to localize the

543 seizure focus. Electrode placement was determined by the surgeons based on the clinical need of

544 each patient.

545 Data were recorded at the Epilepsy Monitoring Unit of the Hospital del Mar, Barcelona, Spain. All

546 subjects provided the informed consent to participate in the study in accordance with the ethical

547 committee of the Pompeu Fabra University as well as Hospital del Mar. All iEEG recordings were

548 performed using a standard clinical EEG system (XLTEK, subsidiary of Natus Medical) with a $500 \mathrm{~Hz}$

549 sampling rate. A uni- or bilateral implantation was performed using 12 to 16 intracerebral electrodes

550 (Dixi Médical, Besançon, France; diameter: $0.8 \mathrm{~mm}$; 5 to 15 contacts, $2 \mathrm{~mm}$ long, $1.5 \mathrm{~mm}$ apart) that

551 were stereotactically inserted using robotic guidance (ROSA, Medtech Surgical, Inc).

552

553 To identify the anatomical position of the electrode contacts we used the 3D Slicer software(66).

554 With the registration tool, we coregistered (rigid body, 6 degrees of freedom) the post-implantation

555 CT scan to the pre-implantation MRI. We then added the electrode fiducials on a glass model of each

556 patient's brain obtained with the segmentation tool of the Freesurfer bundle (67). To obtain a single

557 model we coregistered all studies on the MNI152 template provided by the Freesurfer bundle using a

558 semi-automated registration process of 3D Slicer. Briefly, we calculated a linear transform with 12

559 degrees of freedom by superposing and morphing each patient's brain MRI onto the MNI brain 
560 template, then we used the transform matrix to translate, shift, skew and resize all other studies (CT

561 scan, and unaltered MRI) accordingly. Since the 3D Slicer interface shows the MNI coordinates when

562 hovering the mouse pointer, we could identify structures touched by electrode contacts both by

563 visual inspection and by referring to the aforementioned coordinates.

564

565

\section{Behavioral task}

568 The behavioral task was a variation of the standard Serial Reaction Time Task (SRTT), a type of 569 paradigm that promotes automation of sequential motor behavior (17). Differently from the original 570 task, however, here, in a small subset of trials, the sequential automated action was occasionally 571 interrupted by a cue that required the subjects to switch to a different goal instructed at the 572 beginning of the experiment.

573 The task comprised a maximum of 500 experimental trials preceded by 60 trials of training. There 574 were two types of trials: automatic and switch. Every trial started with a waiting period of $700 \mathrm{~ms}+-$ $575200 \mathrm{~ms}$ during which the screen remained blank. After this, subjects were presented with a virtual 4 576 by 4 square keyboard.

577 During automatic trials, a sequence of five keys was highlighted sequentially (green cue) upon

578 button-press. Subjects were instructed to press the cued key as rapidly as possible until the end of 579 the sequence. Each trial terminated at the end of the sequence, and the following one started. The 580 sequence was pseudorandomly generated at the beginning of the experiment to respect a spatial 581 uniform distribution over the keyboard and it was maintained constant throughout the experiment. 582 Switch trials started with the same highlighted key as the automatic trials (green cue), and the next 583 step in the sequence was highlighted upon a button press. Differently from automatic trials, however, 584 one of the intermediate steps of the sequence (i.e. step 2-4 selected at random) highlighted in red 585 (switch cue). Upon presentation of the switch cue, subjects were required to halt the ongoing 586 sequence of movements as fast as possible and press an alternative, uncued key. Participants 587 received all the instructions prior to the beginning of the experiment. Feedback was provided for 588 neither the correct nor incorrect performance. The training phase only comprised automatic trials, 589 whereas the experimental phase included a combination of automatic and switch trials pseudo590 randomly interspersed every $7+-2$ trials.

591 The experimental setup ran on a portable capacitive screen fixed to the hospital overbed table. The 592 tablet included a custom-made Java-based application running the experimental task and logged 593 behavioral performance at $50 \mathrm{~Hz}$ whereas task synchronization with the neural recordings was 594 achieved through serial communication with the recording system. Subjects sat in a comfortable 595 position that avoided motor constraints to the arm. After receiving the instructions, subjects 
596 underwent a short session that exemplified the task. After this, the experimental session started. Subjects could withdraw at any point during the task.

598

599

602 All electrophysiological data were preprocessed in Matlab (EEGLAB toolbox) and subsequently 603 analyzed in Python using custom scripts based on the Numpy, Scipy, SkLearn and MNE libraries.

604 Data were initially filtered using a two-way zero phase-lag, FIR bandpass filter (2-200 HZ) and an 605 additional notch filter (window $=2 \mathrm{~Hz}$ ) at $50 \mathrm{~Hz}, 100 \mathrm{~Hz}$ and $150 \mathrm{~Hz}$ to remove AC current 606 contamination and respective harmonics. Following this step, the signals were individually re607 referenced to the average potential of all electrodes for each subject.

608 After filtering, artifacts derived from strong muscle activity or interference due to contact with 609 electrical devices were identified by visual inspection and respective epochs rejected. To reduce 610 remaining artifacts (i.e. cardiac artifacts, muscle twitches), we applied a combination of Principal 611 Component Analysis (PCA) and independent component analysis (ICA). In brief, we performed PCA 612 on all channels and identified those components which accounted for $>98 \%$ of the variance. Such 613 components were subsequently decomposed into the same number of independent components 614 through ICA. At this point, each component time series was visually inspected and components that 615 reflected signal artifacts were rejected. The selection of artifact components was based on a careful 616 inspection of their power spectrum, correlation with other physiological measures (i.e. ECG), and the 617 relation to the temporal structure of the experiment. The rejection was performed by setting the ICA

618 weight associated to the artifact component to 0 . The signal was further reconstructed by inverting 619 the ICA operation and the subsequent PCA operation after having renormalized the remaining weights.

\section{Amplitude analysis}

625 For each subject, the filtered and artifact-free signal was split into epochs according to the trial 626 structure of the task. Each epoch was individually baseline corrected by subtracting the mean 627 amplitude value in a temporal window of $500 \mathrm{~ms}$ preceding the beginning of each trial. To identify 628 task-selective channels displaying changes in the amplitude of the signal (i.e. Movement Related 629 Cortical Potentials (MRCP)) we extracted a set of 3 descriptors (absolute mean, variance and 630 integral) and assigned binary labels to each epoch according to the trial type (0=automatic, 631 1=switch). Further, we applied a classification method based on the Linear Discriminant Analysis 632 (LDA) (68). 100 cross-validation steps were performed to assess performance with Fishers F1 score 
633 on class-balanced bootstraps of data samples ( $80 \%$ training, $20 \%$ testing). The channels providing

634 the highest classification accuracy were finally selected as the task-related channels. Note that this

635 analysis was naive with regards to the electrode location or the polarity of the event. This step

636 allowed us to narrow down our analysis to those contact points that displayed a task-related change

637 in the amplitude (a detectable difference between conditions) for each subject.

638 Spectral analysis revealed the presence of MRCPs in the low-frequency range between 1 and $2 \mathrm{~Hz}$

639 (not shown). Trial-by-trial MRCP peaks in the switch condition were therefore identified by low-

640 passing the signal up to $2 \mathrm{~Hz}$ using a two-ways zero-phase FIR filter and applying a peak detection

641 algorithm that estimated the time of the absolute peak amplitude in the interval between stimulus

642 presentation (switch-cue) and the response. Single-trial stimulus-peak interval, as well as peak-

643 response interval, were further calculated by subtracting the stimulus presentation time from the

644 peak time and the peak time from the response time respectively.

645 Finally, the statistical analysis of amplitude differences was performed through a T-statistics one-

646 dimensional non-parametric cluster based permutation test (30) as implemented in the MNE toolbox

647 with cluster significance threshold $=0.05$ and number of permutations $=1000$.

\section{Spectral Analysis}

651

652 Spectral analyses were performed using a DPSS multi-taper method $(69,70)$ as implemented in the

653 MNE toolbox. Trials were aligned to the relative MRCPs peak time, rather than to the behavioral

654 response, in order to avoid artifacts due to the averaging temporally variable signal (19). Changes in

655 the power with respect to the baseline where computed by z-transforming the power spectrum.

656 Statistical differences in the time-frequency power between conditions were calculated through T-

657 statistics two-dimensional cluster based permutation analysis as implemented in the MNE toolbox

658 setting cluster significance threshold $=0.05$ and number of permutations $=1000(30)$.

\section{Inter-trial phase coherence (ITPC)}

662

663 We estimated inter-trial phase coherence to quantify the frequency-dependent synchronization

664 across MRCP peak-aligned trials through Phase Locking Value (PLV) method (71). ITCP is computed

665 as:

666

$$
I T P C=\frac{1}{N}\left|\sum_{n=1}^{N} e^{j \phi_{n}}\right|
$$


668 where $N$ is the number of trials in one condition and $\phi$ represents the phase estimate at the $n^{\text {th }}$ trial.

669 ITCP is bounded between 0 and 1 , where 1 represents full phase synchronization. In order to test

670 differences in ITCP between conditions, we used the cluster-based permutations method proposed

671 by (30). First, we applied a z-transform to the difference in coherence between conditions ( $\mathrm{Z}_{\text {ITPC }}$ ) that

672 rendered the distribution approximately normal (72):

673

$$
Z_{\text {ITPC }}=\frac{\left(\tanh ^{-1}\left(\left|I T P C_{1}\right|\right)-\left(1 / d f_{1}-2\right)\right)-\left(\tanh ^{-1}\left(\left|I T P C_{2}\right|\right)-\left(1 / d f_{2}-2\right)\right)}{\sqrt{\left(1 / d f_{1}-2\right)+\left(1 / d f_{2}-2\right)}}
$$

676 Where $I T P C_{\mathrm{i}}$ and $d f_{i}$ represent the inter-trial phase coherence and degrees of freedom for the $i^{\text {th }}$ 677 condition respectively. To account for the positive bias of ITPC, we used the same amount of trials for 678 the two conditions compared. Second, we selected those regions where $\mathrm{z}>2.58$ corresponding to the 679 99th percentile of the distribution. Finally, we assessed the significance of the measured difference 680 against the $\mathrm{HO}$ obtained by computing the coherence difference between surrogate groups 681 constructed by permuting 1000 times the original labels and extracting the resulting Montecarlo $\mathrm{P}$ 682 value.

\section{Single trial ITPC}

ITPC is by definition an average measure across multiple trials. An estimate of the contribution of the single trial to the average ITPC (STPC), however, can be obtained by computing the difference between the ITPC across all trials and the ITPC across all but one trial following the method proposed by (23) and previously applied by (22). The Single Trial ITPC $\left(S T P C_{i}\right)$ for the $i^{\text {th }}$ trial is computed as follows:

692

$$
S T P C_{i}=N Z_{i t p c}^{\text {all }}-(N-1) Z_{i t p c}^{\text {all-i }}
$$

694 where $N$ is the number of trials and $Z_{i t p c}^{a l l}$ and $Z_{i t p c}^{a l l-1}$ are the z-transformed ITPCs for all trials and all 695 but the $i^{\text {th }}$ trial respectively. Finally, we computed the STPC for each trial and time-frequency bin.

696 The correlation coefficient between STPC and single trial reaction times was calculated using the 697 Spearman's R, as the STPC distribution was found to violate the normality assumption. This 698 operation was repeated in order to cover the whole time-frequency range, resulting in an R map of 699 size time - frequency. 
700 To further compute the statistical significance of the obtained R map and to correct for multiple

701 comparisons we applied cluster permutation analysis for each subject. In brief, for each bin we

702 randomly shuffled the data on both the reaction time and the STPC dimensions and recomputed at

703 each permutation the Spearman's R for a total of 1000 permutations. For each bin we obtained a

704 distribution of $\mathrm{R}$ under random condition, which served to set the threshold of significance to the

$70599 \%$ percentile of the distribution, corresponding to a $\mathrm{p}$ value of 0.01 . Further, each randomly

706 obtained R map was thresholded according to the corresponding significant value, so to obtain a

707 number of time-frequency clusters where the correlation coefficient was found significant. To

708 determine whether the thresholded time-frequency clusters obtained from the experimental

709 condition could be considered statistically significant we compared their magnitude with the ones

710 resulted from the permutation analysis. To this end, for each cluster we integrated the absolute $\mathrm{R}$

711 value so to obtain one magnitude value per cluster. Further we computed the distribution of cluster

712 magnitudes under random condition and calculated its $99 \%$ percentile, corresponding to a p value of

713 0.01. Cluster magnitudes in the experimental condition that exceeded this threshold were considered

714 statistically significant. Whole brain maps were obtained by projecting the average $\mathrm{z}$ score of those

715 individual contact points showing a significant cluster in the time window between -0.6 and $0.4 \mathrm{~s}$ in

716 the MNI space.

717

718 Phase-amplitude coupling (PAC)

719

720 PAC is a measure that quantifies the modulatory effect of low-frequency phase on higher frequency 721 amplitude as a signature of the interaction between their underlying processes resonating at 722 different frequency bands. PAC was computed through the Generalized Linear Models (GLM) method

723 (37) that captures the proportion of variance explained by an underlying linear relationship between

724 analytical amplitude (i.e. envelope, modulated) and phase (modulating) as obtained by Hilbert

725 transforming the signal, using the PACpy toolbox (https://github.com/voytekresearch/pacpy).

726 We restricted our analysis of PAC to the ROIs emerged from cluster-based permutation analysis and

727 selected as modulatory frequency band the significant frequency domain range for each subject. Our

728 epoch selection was also restricted to the temporal window of approximately $400 \mathrm{~ms}$ where a

729 significant increase in phase alignment was detected. For each subject, we obtained one surrogate

730 signal for fast and slow trials by concatenating the respective single trial windows, so to achieve the

731 temporal resolution necessary for this type of analysis. Further, we computed PAC values between

732 the selected modulatory phase and the amplitude of higher frequencies $(10-100 \mathrm{~Hz}$ in steps of $2 \mathrm{~Hz}$,

733 modulated frequency), and obtained the difference between the two conditions.

734 Statistical significance between the two conditions was tested through z-statistics against the null-

735 hypothesis of samples from both conditions belonging to the same distribution. This was obtained by

736 randomly permuting the conditions' labels and calculating the 95 percentile of the maximum PAC 
737 value achieved under the assumption that the two conditions were sampled from the same

738 distribution (38). Note that this approach could introduce spurious oscillations as an artifact due to

739 the concatenation of several signals, where the frequency of the oscillation is directly proportional to

740 the length of the segments concatenated. We control for this possibility by choosing temporal

741 windows that may introduce artifacts at lower frequencies than those considered in this analysis. In

742 addition, the same concatenation is applied equally for both conditions and therefore it is unlikely to

743 affect the comparison.

744

745

746

\section{ACKNOWLEDGMENTS}

747

748 We thank the patients at the Hospital del Mar - Epilepsy Unit for participating as subjects in the study

749 and the Hospital del Mar - Epilepsy Unit staff for providing valuable technical support during the

750 experiments. This work was supported by the H2020 Research and Innovation grant "Virtual Brain

751 Cloud" (826421). RR and RZ were supported by the project "Clúster Emergent del Cervell Humà"

752 (CECH) ref. 001-P-001682. ATC was supported by the Bial Foundation grant 106/18.

753

754

755

756

\section{COMPETING INTERESTS}

757

758 The authors declare no competing interests

759

760

REFERENCES

761

762 1. Rushworth MFS, Hadland KA. Role of the Human Medial Frontal Cortex in Task Switching: A Combined fMRI and TMS Study. J .... 2002;2577-92.

764 2. Rushworth MFS, Walton ME, Kennerley SW, Bannerman DM. Action sets and decisions in the

765 medial frontal cortex. Trends Cogn Sci. Thieme; 2004 Sep;8(9):410-7.

766 3. Nachev P, Kennard C, Husain M. Functional role of the supplementary and pre-supplementary

767 motor areas. Nat Rev Neurosci. 2008;9(11):856-69.

768 4. Hikosaka 0, Isoda M. Switching from automatic to controlled behavior: cortico-basal ganglia

769 mechanisms. Trends Cogn Sci. Elsevier Ltd; 2010;14(4):154-61.

$770 \quad$ 5. Sakai K. Task Set and Prefrontal Cortex. Annu Rev Neurosci. Annual Reviews ; 2008 Jul

$771 \quad 17 ; 31(1): 219-45$.

772 6. Isoda M, Hikosaka O. Switching from automatic to controlled action by monkey medial frontal 773 cortex. Nat Neurosci. Nature Publishing Group; 2007 Feb;10(2):240-8. 
$7747 . \quad$ Roberts RE, Husain M. A dissociation between stopping and switching actions following a lesion of the pre-supplementary motor area. Cortex. Elsevier Ltd; 2015;63:184-95.

776 8. Cohen MX, Cavanagh JF. Single-trial regression elucidates the role of prefrontal theta oscillations in response conflict. Front Psychol. 2011;2(FEB):1-12.

778 9. Cavanagh JF, Frank MJ. Frontal theta as a mechanism for cognitive control. Trends Cogn Sci.

$779 \quad$ Elsevier Ltd; 2014;18(8):414-21.

780 10. Narayanan NS, Cavanagh JF, Frank MJ, Laubach M. Common medial frontal mechanisms of adaptive control in humans and rodents. Nat Neurosci. Nature Publishing Group; 2013;16(12):1888-95.

11. Helfrich RF, Knight RT. Oscillatory Dynamics of Prefrontal Cognitive Control. Trends Cogn Sci. Elsevier Ltd; 2016;20(12):916-30.

12. Voytek B, Kayser AS, Badre D, Fegen D, Chang EF, Crone NE, et al. Oscillatory dynamics coordinating human frontal networks in support of goal maintenance. Nat Neurosci. Nature Publishing Group; 2015;18(9):1318-24.

13. Smith EH, Horga G, Yates MJ, Mikell CB, Banks GP, Pathak YJ, et al. Widespread temporal coding of cognitive control in the human prefrontal cortex. Nat Neurosci. Nature Publishing Group; 2019 Nov 30;22(11):1883-91.

14. Sweeney-Reed CM, Zaehle T, Voges J, Schmitt FC, Buentjen L, Kopitzki K, et al. Thalamic theta phase alignment predicts human memory formation and anterior thalamic cross-frequency coupling. Elife. eLife Sciences Publications Limited; 2015 May 20;4:e07578. in Phase-Amplitude Coupling Facilitate Spatial Attention Control in Fronto-Parietal Cortex. PLoS Biol. 2014;12(8).

16. Phillips JM, Vinck M, Everling S, Womelsdorf T. A long-range fronto-parietal 5- to $10-\mathrm{Hz}$ network predicts "top-down" controlled guidance in a task-switch paradigm. Cereb Cortex. 2014;24(8):1996-2008.

17. Nissen MJ, Bullemer P. Attentional requirements of learning: Evidence from performance

802 18. Hallett M. Movement-related cortical potentials. Electromyogr Clin Neurophysiol. 1994;34(1):5-13.

19. van Diepen RM, Mazaheri A. The Caveats of observing Inter-Trial Phase-Coherence in Cognitive Neuroscience. Sci Rep. Nature Publishing Group; 2018 Dec 14;8(1):2990. waves and trial averaging: The nature of single-trial and averaged brain responses in largescale cortical signals. Neuroimage. 2013 Jun;73:95-112. 
811 Traveling Waves in the Human Neocortex. Neuron. Elsevier; 2018;98(6):1269-1281.e4.

812 22. Hipp JF, Engel AK, Siegel M. Oscillatory Synchronization in Large-Scale Cortical Networks

813 Predicts Perception. Neuron. 2011 Jan 27;69(2):387-96.

814 23. Jarvis MR, Mitra PP. Sampling properties of the spectrum and coherency of sequences of

815 action potentials. Neural Comput. 2001 Apr;13(4):717-49.

816 24. Lisman JE, Jensen 0. The $\theta-\gamma$ neural code. Neuron. NIH Public Access; 2013 Mar

$81720 ; 77(6): 1002-16$.

818 25. Lee D. Behavioral context and coherent oscillations in the supplementary motor area. J

819 Neurosci. Society for Neuroscience; 2004 May 5;24(18):4453-9.

820 26. Sakai K, Hikosaka 0, Nakamura K. Emergence of rhythm during motor learning. Trends Cogn

821 Sci. 2004;8(12):547-53.

822 27. Tanji J. Sequential Organization of Multiple Movements: Involvement of Cortical Motor Areas.

823 Annu Rev Neurosci. 2001;24(1):631-51.

824 28. Karimi F, Kofman J, Mrachacz-Kersting N, Farina D, Jiang N. Detection of Movement Related

825 Cortical Potentials from EEG Using Constrained ICA for Brain-Computer Interface

826 Applications. Front Neurosci. Frontiers Media SA; 2017;11:356.

827 29. Lopour BA, Tavassoli A, Fried I, Ringach DL. Coding of Information in the Phase of Local Field

828 Potentials within Human Medial Temporal Lobe. Neuron. Cell Press; 2013 Aug 7;79(3):594-

829606.

830 30. Maris E, Schoffelen J-M, Fries P. Nonparametric statistical testing of coherence differences. J

831 Neurosci Methods. 2007 Jun;163(1):161-75.

832 31. Mansouri FA, Tanaka K, Buckley MJ. Conflict-induced behavioural adjustment: A clue to the

833 executive functions of the prefrontal cortex. Nature Reviews Neuroscience. Nature Publishing

$834 \quad$ Group; 2009. p. 141-52.

835 32. Murty VP, LaBar KS, Adcock RA. Distinct medial temporal networks encode surprise during

836 motivation by reward versus punishment. Neurobiol Learn Mem. Academic Press Inc.; 2016

837 Oct 1;134(Part A):55-64.

838 33. Berkers RMWJ, Klumpers F, Fernández G. Medial prefrontal-hippocampal connectivity during

839 emotional memory encoding predicts individual differences in the loss of associative memory

$840 \quad$ specificity. Neurobiol Learn Mem. Academic Press Inc.; 2016 Oct 1;134:44-54.

841 34. Minxha J, Adolphs R, Fusi S, Mamelak AN, Rutishauser U. Flexible recruitment of memory-

842 based choice representations by the human medial frontal cortex. Science (80- ). American

843 Association for the Advancement of Science; 2020 Jun 26;368(6498).

844 35. Canolty RT, Knight RT. The functional role of cross-frequency coupling. Trends Cogn Sci. 2010

$845 \quad$ Nov;14(11):506-15.

846 36. Hyafil A, Giraud AL, Fontolan L, Gutkin B. Neural Cross-Frequency Coupling: Connecting

847 Architectures, Mechanisms, and Functions. Trends Neurosci. Elsevier Ltd; 2015;38(11):725- 
848

849

850

851

852

853

854

855

856

857

858

859

860

861

862

863

864

865

866

867

868

869

870

871

872

873

874

875

876

877

878

879

880

881

882

883

884

40.

37. Penny WD, Duzel E, Miller KJ, Ojemann JG. Testing for nested oscillation. J Neurosci Methods. 2008 Sep 15;174(1):50-61.

38. Maris E, Oostenveld R. Nonparametric statistical testing of EEG- and MEG-data. J Neurosci Methods. 2007 Aug 15;164(1):177-90.

39. Moisello C, Crupi D, Tunik E, Quartarone A, Bove M, Tononi G, et al. The serial reaction time task revisited: A study on motor sequence learning with an arm-reaching task. Exp Brain Res. 2009;194(1):143-55.

40. Keele SW, Ivry R, Mayr U, Hazeltine E, Heuer H. The cognitive and neural architecture of sequence representation. Psychol Rev. 2003;110(2):316-39.

41. Crone EA, Wendelken C, Donohue SE, Bunge SA. Neural evidence for dissociable components of task-switching. Cereb Cortex. 2006;16(4):475-86.

42. Monsell S. Task switching. Trends Cogn Sci. 2003;7(3):134-40.

43. Buzsáki G, Anastassiou CA, Koch C. The origin of extracellular fields and currents - EEG , ECoG , LFP and spikes. Nature Publishing Group; 2012;13(June):407-20.

44. Sumner P, Nachev P, Morris P, Peters AM, Jackson SR, Kennard C, et al. Human Medial Frontal Cortex Mediates Unconscious Inhibition of Voluntary Action. Neuron. Elsevier Inc.; 2007;54(5):697-711.

45. RUSHWORTH M, WALTON M, KENNERLEY S, BANNERMAN D. Action sets and decisions in the medial frontal cortex. Trends Cogn Sci. 2004 Sep;8(9):410-7.

46. Rushworth MFS, Hadland KA, Paus T, Sipila PK. Role of the Human Medial Frontal Cortex in Task Switching: A Combined fMRI and TMS Study. J Neurophysiol. 2002 May;87(5):2577-92.

47. Kornhuber HH, Deecke L. Hirnpotentialänderungen bei Willkürbewegungen und passiven Bewegungen des Menschen: Bereitschaftspotential und reafferente Potentiale. Pflugers Arch Gesamte Physiol Menschen Tiere. 1965;284(1):1-17.

48. Shibasaki H, Hallett M. What is the Bereitschaftspotential? Clinical Neurophysiology. Clin Neurophysiol; 2006. p. 2341-56.

49. Colebatch SKJJG. Movement-related potentials associated with self-paced, cued and imagined arm movements. 2002;98-107.

50. Schurger A, Mylopoulos M, Rosenthal D. Neural Antecedents of Spontaneous Voluntary Movement: A New Perspective. Trends Cogn Sci. Elsevier; 2016 Feb 1;20(2):77-9.

51. LIBET B, GLEASON CA, WRIGHT EW, PEARL DK. TIME OF CONSCIOUS INTENTION TO ACT IN RELATION TO ONSET OF CEREBRAL ACTIVITY (READINESS-POTENTIAL). Brain. 1983 Sep;106(3):623-42.

52. Schurger A, Sitt JD, Dehaene S. An accumulator model for spontaneous neural activity prior to self-initiated movement. Proc Natl Acad Sci U S A. National Academy of Sciences; 2012 Oct 16;109(42):E2904-13. 
885 53. Delorme A, Westerfield M, Makeig S. Medial Prefrontal Theta Bursts Precede Rapid Motor Responses during Visual Selective Attention. J Neurosci. 2007;27(44):11949-59.

54. Ramchurn A, de Fockert JW, Mason L, Darling S, Bunce D. Intraindividual reaction time variability affects P300 amplitude rather than latency. Front Hum Neurosci. Frontiers; 2014 Jul 29;8:557.

890 55. Swick D, Turken U. Dissociation between conflict detection and error monitoring in the human anterior cingulate cortex. Proc Natl Acad Sci U S A. National Academy of Sciences; 2002 Dec 10;99(25):16354-9.

893

56. Picard N, Strick PL. Motor areas of the medial wall: A review of their location and functional activation. Cerebral Cortex. Cereb Cortex; 1996. p. 342-53.

57. Rizzuto DS, Madsen JR, Bromfield EB, Schulze-Bonhage A, Seelig D, Aschenbrenner-Scheibe R, et al. Reset of human neocortical oscillations during a working memory task. Proc Natl Acad Sci U S A. National Academy of Sciences; 2003 Jun 24;100(13):7931-6.

58. Voloh B, Valiante TA, Everling S, Womelsdorf T. Theta-gamma coordination between anterior cingulate and prefrontal cortex indexes correct attention shifts. Proc Natl Acad Sci U S A. National Academy of Sciences; 2015 Jul 7;112(27):8457-62.

59. Fell J, Axmacher N. The role of phase synchronization in memory processes. Nat Rev Neurosci. 2011 Feb 20;12(2):105-18.

60. Zavala B, Tan H, Ashkan K, Foltynie T, Limousin P, Zrinzo L, et al. Human subthalamic nucleusmedial frontal cortex theta phase coherence is involved in conflict and error related cortical monitoring. Neuroimage. The Authors; 2016;137:178-87.

61. Muller L, Chavane F, Reynolds J, Sejnowski TJ. Cortical travelling waves: mechanisms and computational principles. Nat Rev Neurosci. Nature Publishing Group; 2018 May 22;19(5):255-68.

62. Isoda $\mathrm{M}$, Hikosaka $\mathrm{O}$. Role for subthalamic nucleus neurons in switching from automatic to

911 63. Cohen MX. A neural microcircuit for cognitive conflict detection and signaling. Trends

$912 \quad$ Neurosci. Elsevier Ltd; 2014;37(9):480-90.

913 64. Jasinska AJ. Automatic inhibition and habitual control : alternative views in neuroscience research on response inhibition and inhibitory control INHIBITION CAN BE EITHER A CONTROL PROCESS ( TO OVERRIDE A PREPOTENT RESPONSE TENDENCY) PROCESSES

917 65. Kenner NM, Mumford JA, Hommer RE, Skup M, Leibenluft E, Poldrack RA. Inhibitory motor 918 control in response stopping and response switching. J Neurosci. Society for Neuroscience; 2010 Jun 23;30(25):8512-8.

920 66. Fedorov A, Beichel R, Kalpathy-Cramer J, Finet J, Fillion-Robin J-C, Pujol S, et al. 3D Slicer as an 921 image computing platform for the Quantitative Imaging Network. Magn Reson Imaging. 2012 
$922 \quad$ Nov;30(9):1323-41.

923 67. Fischl B. FreeSurfer. Neuroimage. 2012 Aug 15;62(2):774-81.

924 68. Blankertz B, Lemm S, Treder M, Haufe S, Müller K-R. Single-trial analysis and classification of

925 ERP components - A tutorial. Neuroimage. 2011 May 15;56(2):814-25.

926 69. Mitra PP, Pesaran B. Analysis of Dynamic Brain Imaging Data. Biophys J. 1999 Feb;76(2):691-

927708.

928 70. Thomson DJ. Spectrum estimation and harmonic analysis. Proc IEEE. 1982;70(9):1055-96.

929 71. Lachaux J-P, Rodriguez E, Martinerie J, Varela FJ. Measuring phase synchrony in brain signals.

930 Hum Brain Mapp. John Wiley \& Sons, Ltd; 1999 Jan 1;8(4):194-208.

931 72. Enochson LD, Goodman NR. CeqQ GAUSSIAN APPROXIMATIONS TO THE DISTRIBUTION OF

932 SAMPLE COHERENCE. 1965 Jun.

933 